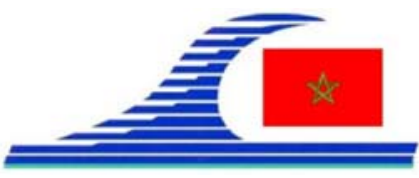

Conférence Méditerranéenne Côtière et Maritime

EDITION 2, TANGER, MAROC (2011)

Coastal and Maritime Mediterranean Conference

Disponible en ligne - http://www.paralia.fr - Available online

\title{
Evolution historique et géomorphologique de la ligne du rivage de la zone côtière de Bou Ahmed (Chefchaouen, Maroc)
}

\author{
Brahim EL MOUTCHOU ${ }^{1}$, Nadia EL MEKADEM ${ }^{1}$, \\ Latifa EL FADEL ${ }^{1}$, Khalil EL HAJJAJI ${ }^{1}$
}

1. Dépt. de Géologie, Faculté des Sciences. BP. 2121, M’hanech. 2. 93000 Tétouan, Maroc. brahimelmoutchou@yahoo.fr

\section{Résumé :}

La ligne de rivage s'établit selon un tracé particulier en fonction de la dynamique régnante dans chaque portion du littoral. La tendance générale de cette dernière, est souvent une tendance de régularisation par redistribution latérale des matériaux. Dans les situations stables, la redistribution se réalise selon un bilan conservant à chaque secteur un "budget" sédimentaire et toute modification de ces échanges aboutit à des perturbations dans l'équilibre local et à l'apparition des phénomènes d'instabilité.

De cette étude, il parait, que l'évolution géomorphologique et morphodynamique de la région de Bou Ahmed, est étroitement liée au contexte géodynamique local de la région et à l'activité anthropique. Sans omettre, les effets des changements climatiques globaux.

\section{Mots-Clés :}

Erosion - Engraissement - Bou Ahmed - Méditerranée - Maroc

\section{Introduction}

L'environnement littoral en général se caractérise par son extrême diversité et par la rapidité de son évolution. C'est un environnement dont l'équilibre dépend à la fois de la nature du substratum, de la quantité des apports sédimentaires et des facteurs dynamiques.

L'étude historique de quelques sites sur le littoral méditerranéen de la région de Bou Ahmed nous a permis, d'appréhender les tendances évolutives et les conditions et facteurs à l'origine de ces tendances.

\section{Caractères généraux de la région de Bou Ahmed}

Située au Nord Ouest du Maroc sur environ 15 km de longueur, le littoral méditerranéen marocain de Bou Ahmed est limité au Nord et à l'Est par la Méditerranée, à l'Ouest et au Sud par la chaîne du Rif (essentiellement les formations paléozoïques de Rif interne), (figure 1).

Les secteurs d'étude font partie intégrante du domaine interne rifain. Il occupe la partie Nord septentrionale du Maroc. Dans l'ensemble c'est un segment de la chaîne alpine. Il 
La connaissance de la Mer :

un vecteur du développement durable en Méditerranée

constitue le tronçon Sud de l'arc de Gibraltar (DIDON et al., 1973 ; DURAND DELGA \& FONTBOTE, 1980). Dans l'ensemble, on note la présence des sebtides et de quelques affleurements des ghomarides et de la dorsale calcaire (KORNPROBST, 1971-74).

Ces unités ont été affectées par une succession de phase tectonique à l'origine des plis de direction SW-NE et SE-NW (CHALOUAN, 1986 ; BENMAKHLOUF, 1990).

Géomorphologiquement, la région présente trois entités morphologiques à savoir : Les plaines basses (Targha et Tighissase), les reliefs élevés (Caps de Zaouia et Aâraben) et les côtes sableuses (Plages s.s).

Le contexte climatique montre un climat de type méditerranéen, marqué par deux saisons distinctes (figure 1) :

- un hiver frais et humide aux précipitations le plus souvent brutales (EL MOUTCHOU 1995 ; EL FADEL, 2006) s'étendant d'Octobre à Mai avec un maximum de pluie en décembre/janvier $\left(\mathrm{P}=126 \mathrm{~mm}\right.$ et $\left.\mathrm{T}=11,9^{\circ} \mathrm{C}\right)$,

- un été chaud et sec, s'étendant de Mai à Octobre avec des températures pouvant atteindre $25^{\circ} \mathrm{C}$ en juillet et août.

Selon l'ABHL (Agence du Bassin Hydraulique du Loukkos/Tétouan), la plupart des stations montent des vitesses moyennes mensuelles des vents inférieures à $15 \mathrm{~km} / \mathrm{h}$ avec une dominance des vents NE et ENE.

L’hydrographie de la région est caractérisée par un réseau hydrographique dense, serré et à régime d'écoulement irrégulier permanent ou temporaire. Dans les zones élevées les oueds ont un régime torrentiel.

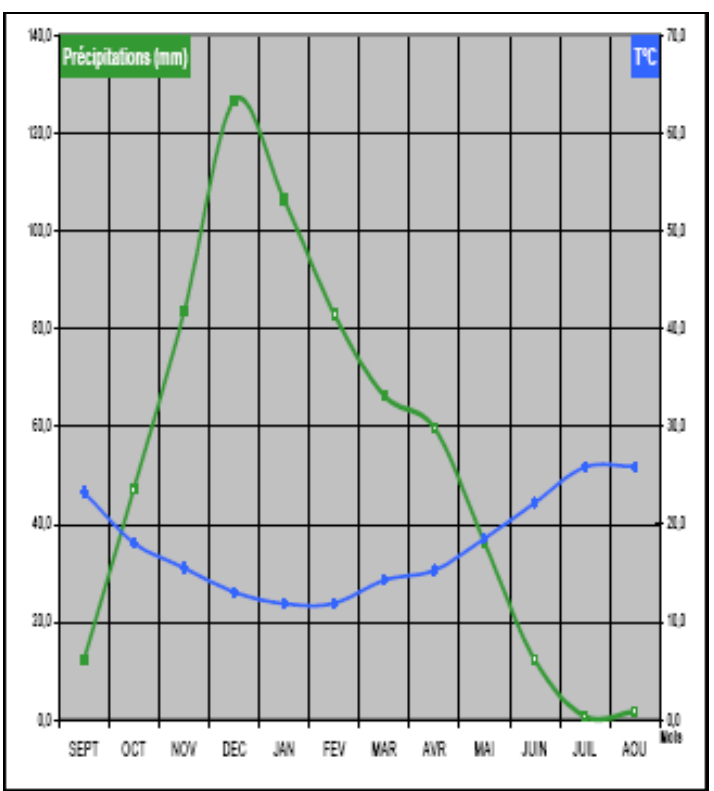

Figure 1. Diagramme ombro-thermique dans de la région de Bou Ahmed. 
Le contexte hydrodynamique montre une dynamique dominée par les effets conjugués des houles et des marées. En général en méditerranée, les marées sont de type semi diurne à marnage faible qui décroît de l'Ouest vers l'Est avec environ $1 \mathrm{~m}$ à Tétouan, $0,90 \mathrm{~m}$ à $\mathrm{Al}$ Hoceima et $0,35 \mathrm{~m}$ à Nador.

Les houles dominantes $\mathrm{E}$ à NE sont en général de faible amplitude. Au voisinage des rivages, elles engendrent deux types de courants : les courants parallèles à la côte et les courants perpendiculaires à la côte (EL MOUTCHOU, 1995 ; EL FADEL, 2006).

\section{Méthodes et techniques d'étude de l'évolution historique}

L'ensemble des documents cartographiques utilisés pour cette analyse se présentent comme suit (tableau.1). Le traitement de l'ensemble de ces documents est effectué en deux étapes :

i. La mosaïque, l'adaptation des échelles et le géoréférencement des photographies aériennes (figures 3 et 4).

ii. la numérisation, la qualification et la quantification des tendances évolutives de la ligne du rivage des différents secteurs du littoral de Bou Ahmed.

Suite à la numérisation de la ligne du rivage, on superpose les différents documents obtenus. Les mesures effectuées nous on permis de calculer les taux d'érosion et/ou d'engraissement issues des différents documents (EL MOUTCHOU, 1995 et 2003 ; GOBERT et al., 1996 ; GAILLOT \& CHAVEROT, 2001 ; EL FADEL, 2006).

\section{Bilan évolutif des littoraux de la région de Bou Ahmed entre 1970 et 2007}

De 1970 et 2007, le littoral méditerranéen marocain de la région de Bou Ahmed montre, les faits évolutifs suivants (figure. 5) :

- des zones en stabilité (Plages de Targha, la partie Nord d'Azenti, El Jaoun et de la partie Sud de Chmaâla jusqu’à Ras Aâraben),

- des zones en érosion (Partie Sud de la plage de Stihat),

- des zones en engraissement (Partie Sud de la plage d'Azenti et la partie Nord de la plage de Chmaâla).

Tableau 1. Documents utilisés dans l'étude de l'évolution historique.

\begin{tabular}{|c|c|c|c|}
\hline Documents utilisés & Echelle & Bandes & Sources \\
\hline $\begin{array}{l}\text { Carte topographique de Bou Ahmed et Talembote de } \\
1970\end{array}$ & $1 / 50000$ & $=====$ & Division de la Carte-Rabat \\
\hline \multirow{4}{*}{ Photographies aériennes (Mission de 1986 - UAM) } & \multirow{4}{*}{$1 / 20000$} & B.18/Ph.45 & \multirow{4}{*}{$U A M-0182$} \\
\hline & & B.19/Ph.52 & \\
\hline & & B.20/Ph.53 & \\
\hline & & B.21/Ph.64 & \\
\hline Image satellite (Prise de 2007) & $1 / 84000$ & $======-$ & Google Earth \\
\hline
\end{tabular}


La connaissance de la Mer :

un vecteur du développement durable en Méditerranée

La synthèse des tendances évolutives du comportement de la ligne du rivage du littoral de la région de Bou Ahmed de 1970 à 2007, présente les faits suivants (tableau. 2).

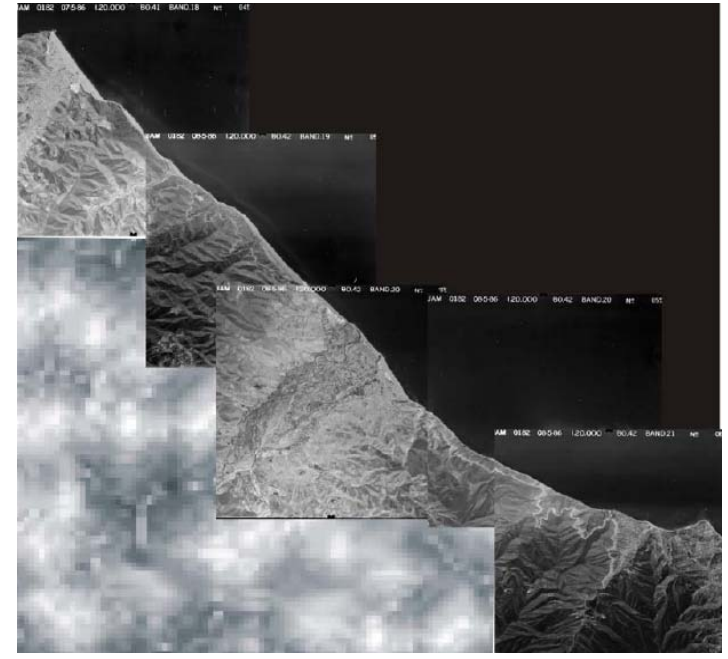

Figure 2. La Mosaïque des photographies aériennes de la région de Bou Ahmed.

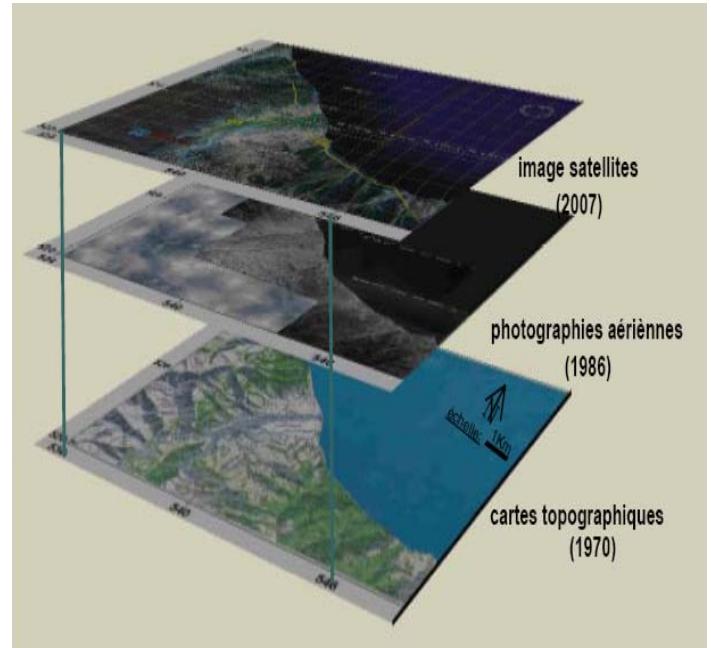

Figure 3. Adaptation des échelles et géoréférencement des documents utilisés.

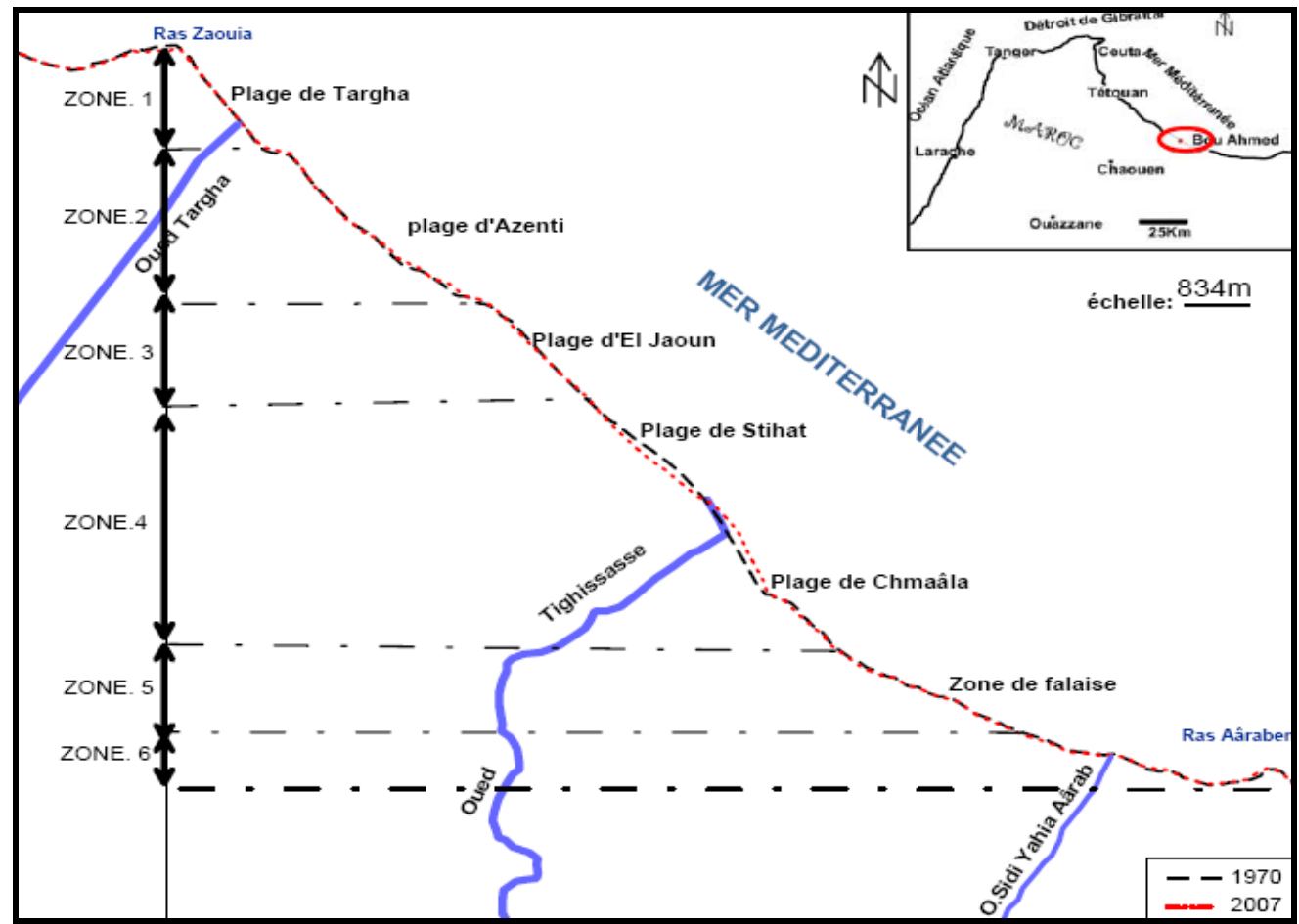

Figure 4. Carte synthétique du comportement de la ligne du rivage en 1970 et 2007. 
Tableau 2. Qualification et quantification de du comportement de la ligne du rivage des littoraux de la région de Bou Ahmed entre 1970 et 2007.

\begin{tabular}{|c|c|c|c|c|c|c|c|c|c|}
\hline & Zone. 1 & Zone. & (en m) & & & one. 4 (en $n$ & & Zone. 5 & Zone. 6 \\
\hline $\begin{array}{l}\text { Taux global } \\
\text { de recul ou } \\
\text { d'avancée }\end{array}$ & 0 & 0 & $+62,5$ & 0 & $-109,4$ & +113 & 0 & 0 & 0 \\
\hline $\begin{array}{l}\text { Taux annuel } \\
\text { de recul ou } \\
\text { d'avancée }\end{array}$ & 0 & 0 & $+01,7$ & 0 & $-03,0$ & $+03,0$ & 0 & 0 & 0 \\
\hline Constats & Stabilité & Stabilité & $\begin{array}{c}\text { Accumula- } \\
\text { tion }\end{array}$ & Stabilité & Erosion & $\begin{array}{c}\text { Accumula- } \\
\text { tion }\end{array}$ & Stabilité & Stabilité & Stabilité \\
\hline Sites & $\begin{array}{r}\text { Plages } d \\
\text { Nord }\end{array}$ & $\begin{array}{l}\text { Targha et } \\
\text { 'Azenti }\end{array}$ & $\begin{array}{c}\text { Plage Sud } \\
\text { Azenti }\end{array}$ & $\begin{array}{c}\text { Plage d'El } \\
\text { Jaoun }\end{array}$ & $\begin{array}{c}\text { Plage } \\
\text { de Stihat }\end{array}$ & $\left|\begin{array}{l}\text { Plage Nord } \\
\text { de Chmaala }\end{array}\right|$ & $\begin{array}{r}\text { Plages } \\
\text { et } S i\end{array}$ & $\begin{array}{l}\text { Sud de C } \\
\text { di Yahia A }\end{array}$ & $\begin{array}{l}\text { maala } \\
\text { rab }\end{array}$ \\
\hline
\end{tabular}

A la lumière de ces résultats, on note l'existence :

- des zones en stabilité avec : Les zones des falaises et les plages ouvertes deTargha, Nord d'Azenti, Sud de Chmaala et Sidi Yahia Aarab,

- des zones en érosion avec : La plage de Stihat,

- des zones en engraissement avec : Les plages Sud d'Azenti et Nord de Chmaala, dues pour l'essentiel, aux apports continentaux et aux transferts de matières latéraux.

Pour une meilleure caractérisation de ces tendances évolutives, une étude détaillée de l'évolution morphologique et tectono - sédimentaire s’avère nécessaire.

\section{Conclusion générale}

De cette étude il ressort que, le littoral méditerranéen marocain de la région de Bou Ahmed, se présente comme un écosystème à équilibre fragile, dominé pour l'essentiel par :

- une dynamique littorale basée sur un système d'échange entre les terres émergées et le milieu marin. Ces échanges dépendent à leur tour du contexte géodynamique local de la chaîne rifaine et du contexte climatique,

- une tendance évolutive marquée, par l'alternance des zones en érosion (Plage de Stihat, avec un taux moyen annuel de recul de la ligne du rivage de l'ordre de -2,95 m/an), des zones en engraissement (Partie Sud de la plage d'Azenti avec un taux moyen annuel de l'ordre de $+1,69 \mathrm{~m} / \mathrm{an}$ et la partie Nord de la plage de Chmaâla avec un taux moyen annuel de l'ordre de $+3,5 \mathrm{~m} / \mathrm{an}$ ) et des zones stabilisées (Zones des falaises et plages ouvertes deTargha, Nord d'Azenti, Sud de Chmaala et Sidi Yahia Aarab). 
La connaissance de la Mer :

un vecteur du développement durable en Méditerranée

\section{Références bibliographiques}

BENMAKHLOUF M. (1990). Genèse et évolution de l'accident de Tétouan et son rôle transformant au niveau du Rif septentrional (Maroc) (depuis l'Oligocène jusqu'à l'actuel). Thèse $3^{\text {ème }}$ cycle, Faculté des Sciences, Rabat, 162 p.

CHALOUAN A. (1986). Les nappes Ghomarides (Rif septentrional Maroc). Un terrain varisque dans la chaîne Alpine. Thèse Doc. Univ Luis Pasteur, Strasbourg, 317 p.

DIDON J., DURAND DELGA M., KORNPROBST J. (1973). Homologies géologiques entre les deux rives du détroit de Gibraltar (Cordillères bétiques occidentalesEspagne). BSGF, (7), XV-2 pp 77-105.

DURAND DELGA M., FONTBOTE J.M. (1980). Le cadre srtructural de la méditerranée occidentale. Colloque C5 "Géologie des chaines alpines issues de la téthys". BSGF, 10, pp 203-226

EL FADEL L. (2006). Evolution morpho - dynamique et analyse morphostructurale du littoral méditerranéen marocain de Oued Laou (Provinces de Tétouan et ChefchaouenMaroc Nord Occidental). Mémoire de DESA. Université Abdelmalek Essaâdi. 76 p.

EL MOUTCHOU B. (1995). Dynamique côtière actuelle et évolution morpho dynamique de la frange littorale entre M'diq et Oued Laou (province de Tétouan, Maroc Nord Occidental). Thèse $3^{\text {ème }}$ cycle, Univ. Mohamed V, Rabat, 165 p.

EL MOUTCHOU B. (2003). Dynamique côtière et évolution spatio- temporelle de la frange littorale méditerranéenne entre Fnideq et Martil (province Tétouan, Maroc). Wokshop. Comm. Intern. Exploration Scientifique de la Mer Méditerranée sous le thème : Erosion littoral en Méditerranée occidentale: Diagnostic et Remèdes. Tanger; Maroc. 18- 21/09/2002, pp 35-39.

GAILLOT S., CHAVEROT S. (2001). Méthode d'étude des littoraux à faible évolution. Cas du delta du Golo (Corse) et du littoral du Touquet (Pas de Calais) en France. Géomorphologie, relief, processus, environnement, Vol. 1, pp 47-54.

GOBERT D., OZER A., CORNET Y. (1996) : Étude diachronique de 1948 à 1990 de l'évolution du littoral des plages de Monza et d'Ablo (cap de Corse) par traitement analogique de photographies aériennes. Photointerprétation, $\mathrm{n}^{\circ}$ 1, pp 49-57.

KORNPROBST J. (1971-74). Contribution à l'étude pétrographique et structurale de la zone interne de Rif (Maroc septentrionale). Not. Mém Serv. géol. Maroc, 251, 256 p. 